\title{
Actitud emprendedora de médicos especialistas en un hospital de Huancavelica, Perú
}

\author{
Jorge Omar Lazo Vera* 1,a; Xavier Alonso Melo Pezo 2,b
}

RESUMEN

Objetivo: Medir la actitud emprendedora de los médicos especialistas en un hospital de Huancavelica, Perú. Determinar si la edad, sexo, tiempo de trabajo, modalidad de contrato, estado civil, ingresos económicos y especialidad influyen en su actitud emprendedora.

Materiales y métodos: Estudio observacional, prospectivo y transversal. Se aplicó un cuestionario calificado a escala de Likert a 32 médicos especialistas sobre actitud emprendedora y también se evaluaron la edad, el sexo, el tiempo de trabajo, el tipo de contrato, el estado civil, los ingresos económicos y la especialidad. Se hicieron análisis descriptivos e inferenciales.

Resultados: El 85 \% mostró una sólida actitud emprendedora, y el 15 \% podría ser emprendedor. Ningún participante descartó el emprendimiento como una opción. En la asociación de la actitud emprendedora y los factores estudiados no se encontraron diferencias significativas con ninguna variable.

Conclusiones: El 85\% de los médicos participantes tienen una actitud positiva hacia el emprendimiento, independientemente de los factores asociados estudiados.

Palabras clave: Necesidades y demandas de servicios de salud; Gestión de la salud poblacional; Economía en atención de salud y organizaciones; Percepción pública de la ciencia (Fuente: DeCS BIREME).

\section{Entrepreneurial attitude of medical specialists at a hospital in Huancavelica, Peru}

\section{ABSTRACT}

Objective: To measure the entrepreneurial attitude of medical specialists at a hospital in Huancavelica, Peru. To determine if age, sex, job tenure, type of contract, marital status, income and medical specialty influence their entrepreneurial attitude.

Materials and methods: An observational, prospective and cross-sectional study. A Likert-scale questionnaire on entrepreneurial attitude was administered to 32 medical specialists. Also age, sex, job tenure, type of contract, marital status, income and medical specialty were assessed. Descriptive and inferential analyses were performed.

Results: Eighty-five percent (85\%) of the study population showed a solid entrepreneurial attitude, while the remaining $15 \%$ might be an entrepreneur. No participant rejected entrepreneurship as an option. Regarding the association between the entrepreneurial attitude and the studied factors, no significant differences were found in any variable.

Conclusions: Eighty-five percent ( $85 \%$ ) of the study population showed a positive attitude towards entrepreneurship regardless of the studied associated factors.

Keywords: Health services needs and demand; Population health management; Health care economics and organizations; Public perception of science (Source: MeSH NLM).

1 Universidad Nacional San Agustín, Facultad de Medicina. Arequipa, Perú.

2 Hospital II Essalud Huancavelica, Servicio de Cirugía General. Huancavelica, Perú.

a Médico especialista en Cirugía Ortopédica y Traumatología, Maestro en Salud Pública.

b Médico especialista en Cirugía General, Maestro en Administración.

*Autor corresponsal. 


\section{INTRODUCCIÓN}

En los últimos veinte años se ha experimentado un gran aumento de nuevos negocios en el país, muchos de ellos están relacionados con personas que pudieron ver una oportunidad en el lugar donde el mercado les presentó una carencia; a ellos se les denomina emprendedores, denominación que en este momento es parte ya del inconsciente colectivo, debido al interés que han tomado, principalmente, las escuelas de negocios, y a su creciente promoción en los medios de comunicación.

Entendemos por emprendedor a un innovador o desarrollador, quien reconoce y se apodera de oportunidades, las convierte en ideas de mercado y les añade valor; además, asume riesgos para poder implementar sus ideas y obtener la compensación a este esfuerzo (1). La actitud emprendedora es poseer la capacidad de iniciar una empresa, un plan o un distinto método de vivir, para lo que se requiere seguridad, perseverancia y decisión de actuar por los propios anhelos (2). Asimismo, el estudio de Alda-Varas et al. la "asocia a una cualidad o actitud vital caracterizada por el despliegue de esfuerzos hacia diferentes tipos de metas, en contextos y organizaciones de naturaleza diversa" (3).

El sector salud no ha sido ajeno a este fenómeno. La aparición de policlínicos, clínicas especializadas y la tercerización son producto de una mayor demanda de atención y los médicos son actores principales, pero ¿serán ellos los beneficiarios directos de este surgimiento?

Es preciso revisar lo ocurrido con la medicina a través de los años. El espíritu empresarial recibió el estímulo de la ambición o del placer del descubrimiento. Muchos avances importantes ocurrieron así, de modo que los responsables eran grandes ambiciosos o grandes exploradores, excepto en circunstancias extremas, como en las epidemias o cuando el profesional actuaba en un entorno de riesgo.

Los médicos con perfil emprendedor son precisamente los que perseveraron ante las amenazas del mercado, bastante saturado y despiadado. El primer paso fue reaccionar para transformar la medicina en una empresa (lo que sería su profesionalización) en un contexto de oportunidad que busca conocer al cliente, identificar sus necesidades y sus deseos, y atenderlo de manera adecuada. La medicina se enfoca como una opción, una coyuntura en proceso de madurez que no busca al profesional que trata la enfermedad, sino al que, finalmente, ofrece salud.

En el sector de la salud peruano, el emprendimiento no ha tenido la relevancia necesaria y no es parte de la formación de futuros profesionales. Con el advenimiento de la cuarta revolución industrial, las oportunidades para nuevos negocios son infinitas, con grandes cambios, retos y oportunidades; pero es necesario prepararnos para asumir estos cambios. Es el médico el llamado a ir en la primera línea de avance, ya que es un emprendedor nato desde que ingresa a la universidad hasta que tiene a su cargo a personal para poder brindar un buen servicio.

Es necesaria la capacidad emprendedora del médico para el logro de nuevas oportunidades, sin dejar de lado los preceptos hipocráticos, este sería el momento de cambiar la visión tradicional de la medicina a una más pragmática de acuerdo con los difíciles tiempos que se avizoran, para tener la posibilidad de transformar proyectos en empresas productivas, rentables y de calidad para mejorar la salud de la población.

Así, Jacobson evalúa el emprendimiento en el sistema de salud pública y resalta que existen innumerables actividades emprendedoras como planeamiento estratégico, eficiencia operacional y generación de recursos, además de un potencial para generar emprendimientos a su favor que por sí solo no sostiene su sistema organizacional hacia la actividad emprendedora (4). Por lo tanto, se hace necesario tener médicos con conocimientos de emprendimiento y enfatizar que el método actual de enseñanza ya no es suficiente para los cambios que se avecinan, no solo para beneficio individual, sino también para el sistema público de salud y en consecuencia, para los propios pacientes ${ }^{(5-7)}$.

La presente investigación constituye un valioso aporte al conocimiento de la intención emprendedora de la población médica, y permite optimizar su enfoque como agente de cambio del sector público y privado. Además, llama la atención hacia un tema que convendría ser implementado en los planes de estudio de las facultades de medicina.

\section{MATERIALES Y MÉTODOS}

\section{Diseño y población de estudio}

Estudio observacional, prospectivo y de corte transversal que se realizó entre mayo y julio de 2019 en el Hospital II EsSalud de Huancavelica, región ubicada en la sierra central del país, a una altitud de $3660 \mathrm{~m} \mathrm{~s}$. n. m. con una temperatura promedio de $9,2{ }^{\circ} \mathrm{C}$ y una población de 454 797 habitantes. La actividad económica está centrada en la minería y la pequeña agricultura. Actualmente, por su contexto social y económico está considerada en situación de extrema pobreza ${ }^{\left({ }^{8}\right)}$. La población de estudio estuvo constituida por los 45 médicos especialistas del Hospital II EsSalud Huancavelica. De los cuales siete no accedieron a participar, cuatro respondieron el cuestionario incompleto y dos estuvieron de vacaciones, por lo que la investigación se realizó con 32 médicos.

\section{Variables y mediciones}

Los datos fueron extraídos de una ficha que registraba las 
variables independientes (edad, sexo, contrato, estado civil, tiempo laboral, especialidad e ingresos económicos) mientras que como variable dependiente se consideró a la actitud emprendedora.

Con base en el instrumento de 17 preguntas, se valoró el deseo, pasión, energía, habilidad para prosperar en incertidumbre, determinación y resistencia, responsabilidad, persuasión, autodisciplina, autoconfianza, responsabilidad social y ética, capacidad de evaluar sistemas de control, capacidad de resolver problemas, contactos en redes, conciencia del mercado, respaldo de necesidades, conocimiento del negocio, y capacidad de rodearse de gente adecuada (9). Para procesar el cuestionario de 17 preguntas a cada una se le asignó un valor entre 1 y 3 y fueron calificadas mediante la escala de Likert ${ }^{(10)}$ y se asignó la puntuación de acuerdo a la tabla 1.

Tabla 1. Instrumento de emprendimiento y educación (validado por Kauffman)

\begin{tabular}{|c|c|}
\hline Actitud & Puntos \\
\hline 1. Eres emprendedor & $40-51$ \\
\hline \multicolumn{2}{|l|}{ 2. Podrías ser } \\
\hline emprendedor & $30-39$ \\
\hline \multicolumn{2}{|l|}{ 3. El emprendimiento } \\
\hline no es una opción & Menos de 29 \\
\hline
\end{tabular}

\section{Análisis estadístico}

Los datos se evaluaron en frecuencia absoluta y relativa. Para la asociación de variables se realizó el análisis bivariado y multivariado (regresión logística) ${ }^{(11-14)}$.

\section{Consideraciones éticas}

Se garantizó la reserva en el manejo de la información suministrada de acuerdo a la declaración de Helsinki ${ }^{(15,16)}$.

\section{RESULTADOS}

Se realizó la encuesta a 32 médicos especialistas y se encontró el grado de actitud "eres emprendedor" en el $85 \%$; el $15 \%$, "podría ser emprendedor”, y ningún participante $(0,00 \%)$ eligió "el emprendimiento no es una opción" (Figura 1).

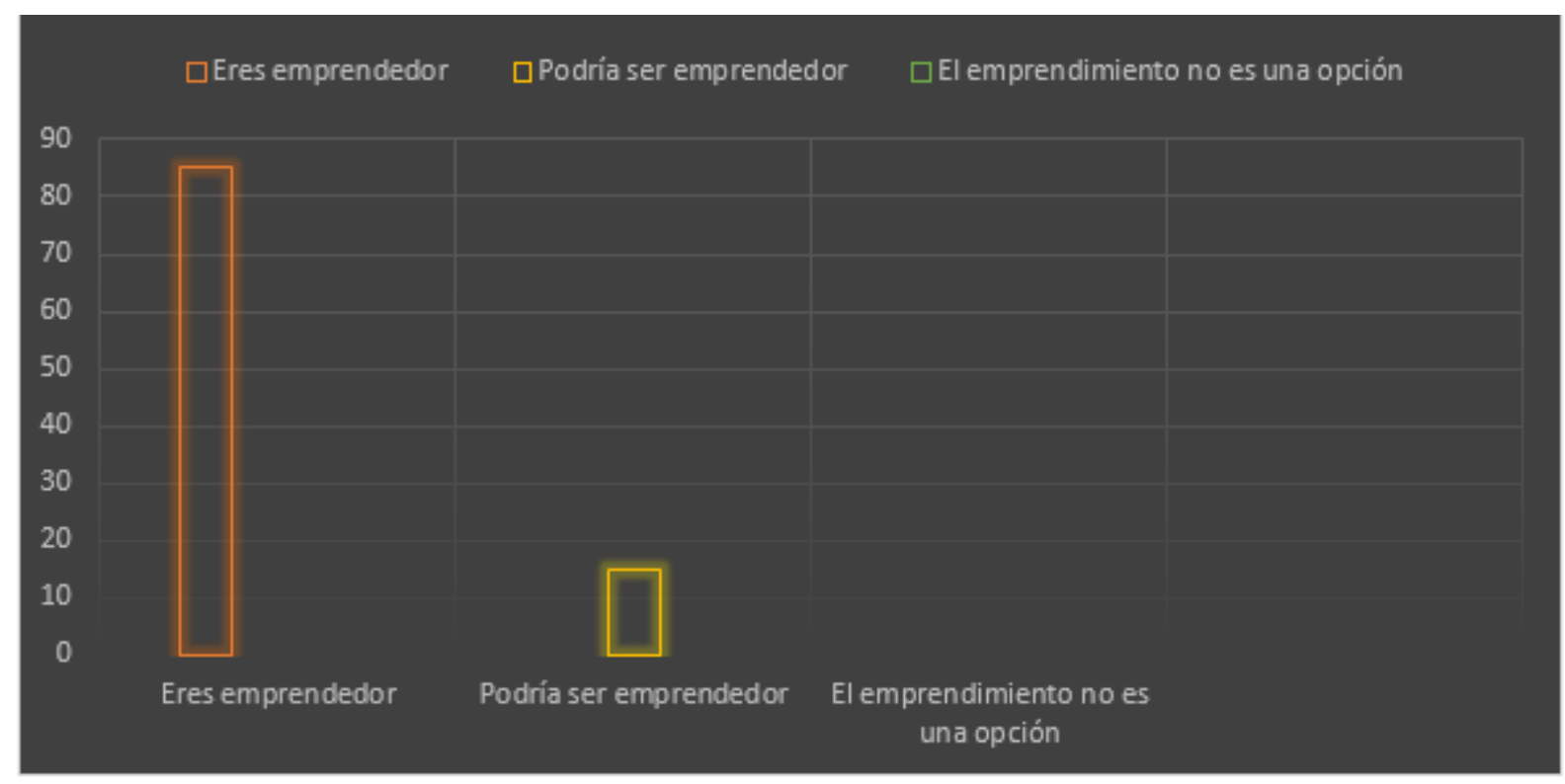

Figura 1. Actitud emprendedora de médicos especialistas (Hospital II EsSalud, Huancavelica) 
El $85 \%$ de los participantes fue de sexo masculino y el $15 \%$, femenino. La mayoría tenían entre 38 y 50 años de edad, con una media de 43 . El $75 \%$ tenía un contrato "estable" (D.L. 728) y el $25 \%$ restante estaba en el sistema de Contrato Administrativo de Servicios (CAS, Ley 1057). La mayoría de los participantes estuvo laborando en el hospital entre dos y doce años, con una media de siete años. Por otro lado, en relación al estado civil, se encontró una mayor frecuencia en el grupo de médicos casados $(56 \%)$. Se determinó una mayor prevalencia de personal médico del área quirúrgica $69 \%$ frente al $31 \%$ del área clínica. El $50 \%$ de los participantes recibía un ingreso promedio de 5000 a 9999 soles; $44 \%$, entre 10000 y 14999 , y el $6 \%$ tenía ingresos mayores a 15000 soles.

Entre la actitud emprendedora y la edad se evidenció una ínfima correlación $(r=0,051)$ además de no encontrarse significancia estadística $(p=0,78)$; mientras que la relación entre el tiempo laboral y la actitud emprendedora no fue significativa $(p=0,43)$. En relación a la actitud emprendedora y el sexo, no se presentó significancia con el sexo masculino $(84,40 \%$ de los participantes) con $p=0,295$ y x $^{2}=1,097$ en la asociación de las variables. No se encontró asociación entre la actitud emprendedora con la modalidad del contrato $(p=0,160)$, el estado civil $(p=0,53)$, los ingresos económicos $\left(p=0,76\right.$ y $\left.x^{2}=0,508\right)$ ni la especialidad médica $\left(p=0,646\right.$ y $\left.x^{2}=0,211\right)$. Además, no se evidenció significancia en la estadística de las variables de estado civil (soltero: $X^{2}=0,29$ y casado: $X^{2}=0,581$ ). Al evaluar la tabla cruzada de ingresos económicos, actitud emprendedora y modalidad de contrato no mostró significancia en su análisis estadístico $\left(x^{2}=0,615\right)$ (Tabla 2).

Tabla 2. Tabla cruzada ingresos económicos, actitud emprendedora y modalidad de contrato

\begin{tabular}{|c|c|c|c|c|c|}
\hline \multirow{2}{*}{\multicolumn{2}{|c|}{ Modalidad de contrato }} & & \multicolumn{2}{|c|}{ Actitud emprendedora } & \multirow[b]{2}{*}{ Total } \\
\hline & & & $\begin{array}{c}\text { Eres } \\
\text { emprendedor }\end{array}$ & $\begin{array}{l}\text { Podría ser } \\
\text { emprendedor }\end{array}$ & \\
\hline \multirow[t]{3}{*}{ Ley 728} & Ingresos económicos & Promedio & 9 & 3 & 12 \\
\hline & & Bueno & 10 & 2 & 12 \\
\hline & Total & & 19 & 5 & 24 \\
\hline \multirow[t]{4}{*}{ CAS } & Ingresos económicos & Promedio & 4 & 0 & 4 \\
\hline & & Bueno & 2 & 0 & 2 \\
\hline & & Muy bueno & 2 & 0 & 2 \\
\hline & Total & & 8 & 0 & 8 \\
\hline \multirow[t]{4}{*}{ Total } & Ingresos económicos & Promedio & 13 & 3 & 16 \\
\hline & & Bueno & 12 & 2 & 14 \\
\hline & & Muy bueno & 2 & 0 & 2 \\
\hline & Total & & 27 & 5 & 32 \\
\hline
\end{tabular}

La asociación de variables de la tabla cruzada ingresos económicos/ actitud emprendedora/ especialidad, no reveló significancia al análisis estadístico (clínicas: $X^{2}=0,287$ y quirúrgicas: $X^{2}=0,707$ ) como se muestra en la tabla 3 .

Tabla 3. Tabla cruzada ingresos económicos, actitud emprendedora y especialidad

\begin{tabular}{|c|c|c|c|c|c|}
\hline & & & \multicolumn{2}{|c|}{ Actitud emprendedora } & \multirow[b]{2}{*}{ Total } \\
\hline \multicolumn{2}{|c|}{ Especialidades } & & $\begin{array}{c}\text { Eres } \\
\text { emprendedor }\end{array}$ & $\begin{array}{l}\text { Podría ser } \\
\text { emprendedor }\end{array}$ & \\
\hline \multirow[t]{3}{*}{ Clínicas } & Ingresos económicos & Promedio & 3 & 2 & 5 \\
\hline & & Bueno & 4 & 0 & 4 \\
\hline & & Muy bueno & 1 & 0 & 1 \\
\hline
\end{tabular}




\begin{tabular}{|c|c|c|c|c|c|}
\hline \multirow{2}{*}{\multicolumn{2}{|c|}{ Especialidades }} & & \multicolumn{2}{|c|}{ Actitud emprendedora } & \multirow[b]{2}{*}{ Tota } \\
\hline & & & $\begin{array}{c}\text { Eres } \\
\text { emprendedor }\end{array}$ & $\begin{array}{l}\text { Podría ser } \\
\text { emprendedor }\end{array}$ & \\
\hline & Total & & 8 & 2 & 10 \\
\hline \multirow[t]{4}{*}{ Quirúrgicas } & Ingresos económicos & Promedio & 10 & 1 & 11 \\
\hline & & Bueno & 8 & 2 & 10 \\
\hline & & Muy bueno & 1 & 0 & 1 \\
\hline & Total & & 19 & 3 & 22 \\
\hline \multirow[t]{4}{*}{ Total } & Ingresos económicos & Promedio & 13 & 3 & 16 \\
\hline & & Bueno & 12 & 2 & 14 \\
\hline & & Muy bueno & 2 & 0 & 2 \\
\hline & Total & & 27 & 5 & 32 \\
\hline
\end{tabular}

En el análisis multivariado se corroboró la falta de asociación entre los diferentes factores sobre su influencia en la actitud emprendedora de los participantes (Tabla 4).

Tabla 4. Análisis multivariado de los factores asociados a actitud emprendedora

\begin{tabular}{|lcc|}
\hline & \multicolumn{2}{c|}{ Coeficientes estandarizados } \\
\hline & Beta & Sig. \\
\hline Edad (años) & 0,216 & 0,470 \\
\hline Sexo & $-0,288$ & 0,149 \\
\hline Tiempo laboral (años) & $-0,028$ & 0,926 \\
\hline Modalidad de contrato & $-0,320$ & 0,193 \\
\hline Estado civil & $-0,415$ & 0,069 \\
\hline Ingresos económicos & 0,006 & 0,977 \\
\hline Especialidad & $-0,105$ & 0,566 \\
\hline
\end{tabular}

Variable dependiente: actitud emprendedora

\section{DISCUSIÓN}

En los participantes del estudio encontramos al grupo "eres emprendedor" con $85 \%$, el $15 \%$ estaba en la clasificación "podría ser emprendedor", y 0 \% en el grupo "el emprendimiento no es una opción".

Nuestra investigación corresponde con la de la fundación Kauffman ${ }^{(9)}$ y otra similar sobre la misma encuesta al público ${ }^{(17)}$ que hallaron que la mayoría fue clasificada dentro del grupo "eres emprendedor" y en las características individuales refirieron necesitar mejorar en los rubros red de negocios, previsión y conciencia de mercado. En nuestros resultados predominaron los aspectos de previsión, responsabilidad social y capacidad de resolución de problemas. Este dato se explicaría si consideramos que los galenos, desde el momento de dedicarse a la medicina entran en un proceso riguroso de selección, una carrera de largo aliento, desarrollo de valores, habilidades y destrezas para el trabajo en equipo; y de ahí en adelante, por un continuo aprendizaje, cualidades que hacen de ellos emprendedores innatos. Relacionado a lo anterior, el director del proyecto GUESSS (Global University 
Entrepreneurial Spirit Student's Survey), en su reporte global y la participación de 208000 estudiantes en 3000 universidades de 54 países, evalúa su intención emprendedora y demuestra que el $9 \%$ de estudiantes intentaría iniciar un emprendimiento luego de culminar sus estudios y el $34 \%$ planea un emprendimiento a los cinco años de egresar. Un patrón central de los encuestados fue tener primero un trabajo como dependiente, y luego emprender un proyecto propio ${ }^{(18)}$.

De la distribución por sexo, el 85 \% fueron varones; y el $15 \%$, mujeres. La investigación de Taype-Rondán et al. (19) estudió una muestra representativa de médicos peruanos y encontró que la proporción entre hombres y mujeres especialistas era de 3,50 a 1 ; frente a 5,60 a 1 del personal médico del hospital. Podríamos inferir que las difíciles condiciones climáticas y de transporte hacen que el personal masculino sea predominante en esta región; además, debido a que la región Huancavelica es una de las más pobres del país ${ }^{(8)}$, para el personal femenino no sería a atractivo trabajar en esta zona.

Respecto a la edad de los participantes de nuestra investigación, el 59 \% se ubica entre los 38 a los 50 años, con una media de 44,37 años, lo que difiere del promedio de edad de los médicos especialistas a nivel nacional, que tiene una distribución homogénea ${ }^{(18)}$. Inferimos que por la ubicación geográfica de este hospital y por las dificultades que presenta esta zona, se ofrecerían más oportunidades para médicos especialistas jóvenes.

El $75 \%$ de los participantes tenía un contrato a plazo indeterminado; y el $25 \%$, un contrato administrativo de servicios (CAS), situación similar a la de los trabajadores de EsSalud ${ }^{(20)}$.

En relación al personal distribuido según el tiempo laboral, el $75 \%$ tenía entre tres y doce años de servicio, similar al $65 \%$ del personal médico de EsSalud que se encuentra en el mismo grupo etario ${ }^{(20)}$. De ello podemos colegir que el aumento demográfico de la población peruana iría en paralelo con el aumento de personal médico encargado de atender a esta población.

En cuanto al estado civil, encontramos que el $56 \%$ de los participantes están casados; $21 \%$, solteros; $16 \%$, convivientes; y $7 \%$ son divorciados. Este resultado difiere del promedio de la población peruana, donde los solteros son $38,50 \%$; los casados representan el $25,70 \%$, y los convivientes, $26,70 \%{ }^{(21,22)}$. Lo anterior se podría explicar por el mayoritario grupo etario de 38-50 años que labora en ese nosocomio.

Respecto a la especialidad de los médicos, el mayor porcentaje corresponde a personal de cirugía 69 \%; Taype-Rondán et al. (19) reportaron una proporción de 1,30 a 1 entre especialistas del área quirúrgica, comparado con el área clínica.

De los ingresos económicos, se tomó como promedio el rango entre 5000 a 10000 soles, y encontramos que el $50 \%$ de los participantes tenían "buenos" ingresos, el $44 \%$ tuvo ingresos promedio, y el $6 \%$, "muy buenos". El reporte de Taype-Rondán et al. demostró que $2 / 3$ de los médicos especialistas peruanos obtuvieron ingresos mayores a los 5000 soles, mientras que el $1 / 3$ registró ingresos menores ${ }^{(19)}$.

Como una limitación del estudio podemos señalar que en la búsqueda bibliográfica de investigaciones relacionadas al tema se encontraron aquellas que evalúan la actitud emprendedora o perfil ce emprendimiento en estudiantes de ciencias de la salud ${ }^{(4-5,16,23)}$, no encontramos trabajos similares realizados en médicos especialistas.

En conclusión, el $85 \%$ de médicos tuvo una fuerte actitud emprendedora y el $15 \%$, potencial emprendedor; por lo tanto, el $100 \%$ de los participantes presentaron predisposición al emprendimiento. En el análisis bivariado, la asociación entre actitud emprendedora y el sexo, la modalidad de contrato, el estado civil, los ingresos económicos y la especialidad no mostró significancia estadística. Al análisis multivariado, con la actitud emprendedora como variable dependiente y el sexo, edad, tiempo laboral, modalidad de contrato, estado civil, ingresos económicos y especialidad de los participantes como variables independientes, no se encontró asociación al $95 \%$ de nivel de significancia.

Contribuciones de autoría: Xavier Alonso Melo Pezo y Jorge Omar Lazo Vera participaron de la concepción del trabajo de investigación, diseño, recolección de datos, análisis, interpretación de datos, revisión crítica y redacción del artículo.

Fuentes de financiamiento: Este artículo ha sido financiado por los autores.

Conflictos de interés: Los autores declaran no tener ningún conflicto de interés.

\section{REFERENCIAS BIBLIOGRÁFICAS}

1. Kuratko DF. Entrepreneurship: theory, process, and practice. 2da ed. California Thomson South Western; 2006.

2. Mas J. Asesoría estratégica del retail. Disponible en: https:// www.crearmas.com/wp-content/uploads/2019/01/actitudemprendedora.pdf

3. Alda-Varas R, Villardón-Gallego L, Elexpuru-Albizuri I. Propuesta y validación de un perfil de competencias de la persona emprendedora. Implicaciones para la formación. Rev Electron Investig Psicoeduc Psicopedag. 2012; 10(3): 1057-80.

4. Jacobson PD, Wasserman J, Wu HW, Lauer JR. Assessing entrepreneurship in governmental public health. Am J Public Health. 2015; 105(Suppl. 2): S318-22. 
5. Ahmad H, Akram N. Should medical entrepreneurship be included in the undergraduate curriculum? A literatura review. British J Healthcare Manag. 2018; 24(1): 40-4.

6. Niccum BA, Sarker A, Wolf SJ, Trowbridge MJ. Innovation and entrepreneurship programs in US medical education: a landscape review and thematic analysis. Med Educ Online. 2017; 22(1): 1360722.

7. Krauss C, Franco JP, Bonomo A, Mandirola N, Platas AL. Entrepreneurial intention of health sciences' students in the Catholic University of Uruguay. Enfermería: Cuidados Humanizados. 2018; 7(1): 102-14.

8. Colaboradores de Wikipedia. Departamento de Huancavelica [Internet]. 2020. Disponible en: https://es.wikipedia.org/wiki/ Departamento_de_Huancavelica\#Ubicaci\%C3\%B3n

9. Muller A. Are You Entrepreneur? Kauffman FastTrac. Entrepreneurial Characteristics Survey. Kauffman Foundation; 2012.

10. Martínez L. Escala de Likert: qué es y cómo utilizarla [Internet]. HubSpot. 2019. Disponible en: https://blog.hubspot.es/service/ escala-likert

11. Dawson-Saunders B, Trapp RG. Bioestadística Médica. 2da ed. México: El Manual Moderno; 1997.

12. Polit F, Hungler P. Investigación científica en Ciencias de la Salud. 6ta ed. México: McGraw-Hill; 2000.

13. Hernández Sampieri R, Fernández Collado C, Baptista Lucio MP. Metodología de la investigación. 6ta ed. México: Mac Graw Hill; 2014.

14. Chirinos RM. Metodología de la investigación. 1ra ed. Arequipa: Universidad Nacional de San Agustín; 2012.

15. Manzini JL Declaración de Helsinki: principios éticos para la investigación médica sobre sujetos humanos. Acta Bioethica. 2000; 6(2): 321-34.

16. Gómez Tabares GE, Molina Restrepo ME. Evaluación ética de proyectos de investigación: una experiencia pedagógica Universidad de Antioquia Colombia. Invest Educ Enferm. 2006; 24(1): 68-77.

17. Forbes. What Drives Would-be Entrepreneu? The results are in [Internet]. Missouri: Kauffman Foundation; 2012. Disponible en: https: / / www. forbes.com/sites/kauffman/2012/06/27/what-driveswould-be-entrepreneurs-the-results-are-in/\#3322884b4b57

18. Seiger P, Fueglistaller U, Zellweger T, Braun I. Global Student Entrepreneurship 2018: Insigthts from 54 Countries. Global GUESSS Report. 2018.

19. Taype-Rondán A, Torres-Román S, Herrera-Añazco P, Alva Díaz C, Brañez-Condorena A, Moscoso-Porras MG. Ingresos económicos en médicos peruanos según especialidad: un análisis transversal de la ENSUSALUD 2015. Rev Perú Med Exp Salud Pública. 2017; 34(2): 18391.

20. CONAREME: Consejo Nacional de Residentado Médico. Postulantes con modalidad libre con vínculo laboral con EsSalud [Internet]. Lima: Conareme. 2019. Disponible en: https://www.conareme.org.pe/ web/comunicados-CONAREME.php

21. INEl: Instituto Nacional de Estadística e Informática. Estadísticas de Empleo [Internet]. Lima: INEI. 2018. Disponible en: https://www. inei.gob.pe/estadisticas/indice-tematico/\%20ocupacion-y-vivienda/

22. INEl: Instituto Nacional de Estadística e Informática. Perú: Perfil sociodemográfico - Informe Nacional. Censos Nacionales 2017: XII de Población, VII de Vivienda y III de Comunidades Indígenas. 2017. Disponible en: https://www.inei.gob.pe/media/MenuRecursivo/ publicaciones_digitales/Est/Lib1539/libro.pdf

23. Wu J, Wu L. Evaluation of Medical College Students' entrepreneurial skills and its relationship with social intelligence. Open J Soc Sci. 2019; 7(4): 13-23.
Correspondencia:

Jorge Lazo Vera

Dirección: Villa Médica Torre 6, Dpto.1302 J.L. Bustamante y Rivero. Lima, Perú.

Celular: 959633834

Correo electrónico: jlazov@unsa.edu.pe

Recibido: 02 de junio de 2020

Evaluado: 07 de agosto de 2020

Aprobado: 17 de agosto de 2020

(c) La revista. Publicado por Universidad de San Martín de Porres, Perú. (c) $\mathbf{\text { Br }}$ Licencia de Creative Commons Artículo en acceso abierto bajo términos de Licencia Creative Commons Atribución 4.0 Internacional. (http://creativecommons.org/licenses/by/4.0/)

ORCID iDs

Xavier Alonso Melo Pezo

(- https: / / orcid.org/0000-0001-5818-9051

Jorge Omar Lazo Vera 